\title{
EXACT SOLUTION OF THE OPTICAL RESPONSE OF THICK SLABS IN THE DISCRETE DIPOLE APPROACH
}

\author{
G.P.M. POPPE and C.M.J. WIJERS \\ Twente University, Enschede, The Netherlands
}

Received 18 May 1990

\begin{abstract}
The recently developed double cell technique, which describes the optical response of an arbitrary semi-infinite dielectric crystal taking into account internal field effects, is extended to include the response of thick slabs. The surface sensitivity of the first technique is fully retained. The implications of the internal field effects on the microscopy of these thick slabs are examined for three simple model systems. Further, we investigated under which conditions deviations from classical Fresnel-behaviour are to be expected and how important these corrections are.
\end{abstract}

\section{Introduction}

Transmission of light through thick plates of transparent materials, serving as a substrate, plays an important role in several commonly used spectroscopies, especially those operating in the infrared. This kind of IR-spectroscopy is one of the few spectroscopies, used in practical studies in the field of catalysis and electrochemistry [1-4]. In the experiment one creates changes of this substrate, confined to the outermost layers, like the adsorption of different kinds of gases. The occurring variations in the IR optical transmission have to be interpreted in terms of surface chemical and physical reactions. The quality of such an interpretation depends of course on the quality of the theoretical model used for the interpretation itself. The conditions which have to be imposed upon such a theoretical model, are particularly severe. At the one hand the passage of the light through the substrate needs to be accounted for, being a macroscopic problem; at the other hand the adsorption process needs to be treated correctly, being a microscopic problem. These two conditions look hard to reconcilc. The best solution would be a microscopic one, which is also able to account for the bulk properties of the slab. In this article the so-called thick slab method, which is fully capable of performing that task, will be derived. The thick slab method is a special implementa- tion of the discrete dipole model, which has been developed to describe the optical properties of dielectric systems and has basically only three starting points. This gives the method a high transparency from the physical point of view, but allows nevertheless for a rigorous microscopic description of the problem. The remarkable aspect of the thick slab method is that it yields an exact solution. This in turn should give it a reliability which goes beyond that of other approaches used hitherto (e.g. ref. [2]), where inevitably the aspects of the problem which could not be incorporated theoretically, had to be overcome by quite a lot of assumptions, being often difficult to verify. The thick slab method further employs the idea of the double cell method developed by the authors $[5,6]$. In this double cell method a real space description and a normal mode decomposition have been synthesized successfully to describe the surface reflection problem. In the thick slab method analogously two surface areas are used and in the bulk area of the slab the number of normal modes has been doubled. Although large parts of this approach are of analytical nature, the final solution has to be obtained numerically. Also in this sense the thick slab method turns out to be extremely efficient. In this article the essential theoretical aspects of the thick slab method will be given. From a number of examples applying to very simple slabs of cubic configuration and 
homogeneous composition, some of the more remarkable non-classical aspects of the method will be shown. This involves the existence of a surface zone at both sides of the slab where rapid oscillations (wiggles) in the dipole strength and a long wavelength bulklike interference phenomenon occur. The thick slab method allows for a conceptual separation of the two. The simple slabs which serve as an example, can easily be characterized by the ratio $\alpha / \alpha_{0}$, where $\alpha$ refers to the polarizability belonging to the discrete dipoles of which the system has been composed and $\alpha_{0}$ is a certain characteristic polarizability to be defined later on. This ratio is strongly related to the ratio in which internal and external fields contribute to the local field. For three values of this coupling ratio, the microscopic response and the macroscopic transmission has been studied. As such, an overview has been obtained of how the discrete dipole model behaves for thick slabs.

\section{Optical response of dielectric slabs}

\subsection{Basics and configurational requirements}

As said in the introduction it is the aim of this ariticle to develop a method to calculate exactly the optical response of thick, crystalline dielectric slabs using the discrete dipole model. This method is a direct extension of previous work by the authors in this field, notably the double cell method to treat the easier problem of the optical response of semi-infinite systems $[5,6]$. From this previous work we summarize, prior to treating the thick slab method, the nccessary ingredients. For any dielectric system the optical response can be calculated by means of the discrete dipole model. All that is required for such an approach, is subdivision of the system into a number of cells. Number, shape and size of the cell can in principle be chosen freely, as long as the overall dimensions are small in comparison with the wavelength of light. The cells moreover are not allowed to exchange charge during their interaction with the incident electromagnetic wave as a result of the requirement of dielectric behaviour. (Note that also metals obey this criterion for sufficiently high energies ( $\hbar \omega>$ $1 \mathrm{eV})$ ). The number of atoms cq. molecules in each cell is arbitrary within the limitations given before, but we prefer the smallest number possible. The position in space of the center of the $i$ th cell will be given by $\boldsymbol{r}_{i}$. Optically the cell will be characterized by a polarizability tensor $\boldsymbol{\alpha}_{i}$. Through $\boldsymbol{E}_{l o c, i}$, the local field at $\boldsymbol{r}_{i}$, a dipole strength $\boldsymbol{p}_{i}$ is induced. As long as no additional restrictions are imposed upon the configuration, only three theoretical starting points suffice for the description. The fields generated by the dipoles are most conveniently calculated by means of Hertz-potentials, so:

$$
\begin{aligned}
& Z_{j}(\boldsymbol{r}, t)=\frac{\boldsymbol{p}_{j}}{4 \pi \varepsilon_{0}} \frac{\mathrm{e}^{\mathrm{i}\left(k\left|\boldsymbol{r}-\boldsymbol{r}_{j}\right|-\omega t\right)}}{\left|\boldsymbol{r}-\boldsymbol{r}_{j}\right|}, \\
& \boldsymbol{E}_{j}(\boldsymbol{r}, t)=\left(\nabla \nabla^{\mathrm{T}}-\frac{1}{c^{2}} \frac{\partial^{2}}{\partial t^{2}}\right) Z_{j}(\boldsymbol{r}, t) .
\end{aligned}
$$

$\boldsymbol{p}_{j}$ in turn follows from the principle of induction:

$$
\begin{aligned}
\boldsymbol{p}_{j}\left(\boldsymbol{r}_{j}\right) & =\boldsymbol{\alpha}_{j} \boldsymbol{E}_{\mathrm{loc}, j} \\
& =\boldsymbol{\alpha}_{j}\left(\boldsymbol{E}_{\mathrm{cxt}}\left(\boldsymbol{r}_{j}\right)+\boldsymbol{E}_{\mathrm{dip}}\left(\boldsymbol{r}_{j}\right)\right) .
\end{aligned}
$$

Here $E_{\text {ext }, j}$ represents the incoming light beam at site $r_{j}$ and $E_{\mathrm{dip}, j}$ represents the field from all other sources in the system. Using the principle of superposition and ignoring time dependent factors from here on, we have shown that [7]:

$$
\boldsymbol{E}_{\mathrm{dip}}\left(\boldsymbol{r}_{j}\right)=\left[\left(\nabla \nabla^{\mathrm{T}}+k^{2} \mathbf{1}\right) \sum_{i}^{\prime} \frac{\boldsymbol{p}_{i}}{4 \pi \varepsilon_{0}} \frac{\mathrm{e}^{\mathrm{ik|r-r_{i } |}}}{\left|\boldsymbol{r}-\boldsymbol{r}_{i}\right|}\right]_{r=r_{j}}
$$

Equations (1)-(3) govern the behaviour of arbitrary systems of discrete dipoles. Prior to continuing this treatment we give some useful mathematical conventions, which will be used henceforth in the article:

- the primc on top of the summation symbol has the conventional meaning, i.e. omission of the $i=j$ term,

- given two arbitrary vectors $a$ and $b$, we take for $a^{T} b$ the scalar improduct $a b$ and for $a b^{T}$ the direct product tensor or dyad of the two vec- 
tors. This convention allows for efficient use of the simple but useful identity $\boldsymbol{a}^{\mathrm{T}}\left(\boldsymbol{b} \boldsymbol{c}^{\mathrm{T}}\right)=$ $\left(\boldsymbol{a}^{\mathrm{T}} \boldsymbol{b}\right) \boldsymbol{c}^{\mathrm{T}}$

- for any vector $u=\left(u_{x}, u_{y}, u_{z}\right)$ we define its reflected counterpart $\underline{\boldsymbol{u}}$ through $\underline{\boldsymbol{u}}=\left(u_{x}, u_{y}\right.$, $\left.-u_{z}\right)$.

\subsection{Systems obeying parallel translational symmetry}

In this article only systems will be studied which obey parallel translational symmetry. All dipoles belonging to such systems, can be assigned to planes and, if we choose $(\hat{x}, \hat{y})$ such that any lattice plane lies parallel to the $x y$ plane, we can define any of the sites of plane $j$ as:

$$
\begin{aligned}
\boldsymbol{r}_{j, n m} & =\boldsymbol{r}_{j}+\boldsymbol{s}_{n m} \\
& =\boldsymbol{r}_{j}+n \boldsymbol{s}_{1}+m s_{2} \quad \text { (n, m integers) } .
\end{aligned}
$$

Here all the $s_{n m}$ build a lattice, spanned by the vectors $s_{1}, s_{2}$, which can always be written as:

$s_{1}=a(1,0,0)$,

$s_{2}=a(\alpha, \beta, 0)$.

Basically $a$ is a normalization length which can have any value, but for this article it will be put equal to the bulk lattice constant. A next requirement for the fulfilment of parallel translational symmetry is that the incoming beam has a simple plane wave character:

$\boldsymbol{E}_{\mathrm{ext}}(\boldsymbol{r})=\boldsymbol{E}_{0} \exp (\mathrm{i} \boldsymbol{k r})$,

$\boldsymbol{k}=\left(\boldsymbol{k}_{\|}, k_{z}\right)=\left(k_{x}, k_{y}, k_{z}\right)$,

where $\omega$ represents the frequency and $k$ the wave vector. If $c$ equals the light velocity as usual, the two quantities are linked through $\omega=c k=c|k|$. Under those assumptions it has been shown [7] that a single plane $j$ of discrete dipoles produces an electric field $\boldsymbol{E}$ at position $\boldsymbol{r}$, according to:

$\boldsymbol{E}(\boldsymbol{r})=\boldsymbol{f}_{j}(\boldsymbol{r}) \boldsymbol{p}_{j}$,

$$
\begin{aligned}
& \boldsymbol{f}_{j}(\boldsymbol{r})=\frac{1}{4 \pi \varepsilon_{0}}\left[\left[\nabla \nabla^{\mathrm{T}}+k^{2} \mathbf{1}\right] S_{j}(\boldsymbol{r}, \boldsymbol{k})\right]_{\boldsymbol{r}}, \\
& S_{j}(\boldsymbol{r}, \boldsymbol{k})=\sum_{n, m}^{\prime} \exp \left(\mathrm{i} \boldsymbol{k} s_{n m}\right) \frac{\exp \left(\mathrm{i} k\left|\boldsymbol{r}-\boldsymbol{r}_{j, n m}\right|\right)}{\left|\boldsymbol{r}-\boldsymbol{r}_{j, n m}\right|} .
\end{aligned}
$$

The quantity $S_{j}(r, k)$ is the scalar planar lattice sum, which further on will be referred to as "lattice sum". The prime in (7c) only becomes active if $r$ happens to coincide with one of the $\boldsymbol{r}_{i, n m}$. In that case the lattice sum is of the intraplanar type. The other lattice sums, the interplanar ones, will be differentiated between interplanar far and interplanar near, depending on whether the distance of $\boldsymbol{r}$ to the plane exceeds a certain characteristic length $z_{0}$ (being about $a$ ) or not. Through (7) the entire behaviour of plane $j$ becomes controlled by only one dipole strength $\boldsymbol{p}_{j}$. As discussed in our previous articles, this dipole strength is the characteristic one, the only free variable of plane $j$. Further on indices $i$, $j$ will always refer to those characteristic dipole strengths.

We will not start immediately with the treatment of the thick slab method. First two simpler strategies will be discussed, which have been published before, but which contain the necessary elements from which the thick slab method is composed.

\subsection{Limiting cases}

In sections 2.3.1 and 2.3.2 the two limiting cases, being thin slabs [8] and crystalline bulk [9] will be given. The optical response of thin slabs is treated using a real space approach along the direction of the surface normal, while that of the crystalline bulk is treated using an approach in reciprocal space for the same direction by means of a normal mode decomposition. In all that follows, we will be dealing with a set of layers of dipoles. The coordinate system has already been chosen such that the planes lie parallel to the $x y$-plane, so the normal direction will correspond to $\hat{z}$. All planes will be located in the upper half space $\left(z_{i} \geqslant 0\right)$ and the light beam impinges coming from the lower half space. 


\subsubsection{Optical response of thin slabs}

Combining eqs. (2), (3) and (5) for an arbitrary collection of dipole lattice planes obeying the same parallel translational symmetry, results in the following general description:

$p_{i}=\alpha_{i}\left[E_{\mathrm{ext}}\left(\boldsymbol{r}_{i}\right)+\sum_{j} \boldsymbol{f}_{i j} \boldsymbol{p}_{j}\right]$,

$\boldsymbol{f}_{i j}=\boldsymbol{f}_{j}\left(\boldsymbol{r}_{i}\right)$.

The key component of these equations are the transfer tensors $\boldsymbol{f}_{i j}$, for the calculation of which the lattice sums are required. Calculation of transfer tensors in real space is possible but quite time consuming and not very instructive. If we apply transformations of a type already developed in principle by Ewald [10] to the lattice sums, we get numerically far more efficient (by several decades) and more meaningful expressions. The transformations used by us belong to two classes: those based upon Ewald's three-fold integral transform and those based upon his one-fold integral transform. For far interplanar sums, which are mostly used in our calculations, only the following expression for the transfer tensor, obtained by means of the threefold integral transform [11], is needed:

$$
\begin{aligned}
& \boldsymbol{f}_{i j}=\frac{a}{\pi} \sum_{p, q} \exp \left(\mathrm{i} k_{p q}\left(\boldsymbol{r}_{i}-\boldsymbol{r}_{j}\right)\right) \boldsymbol{d}_{p q}, \quad\left(z_{i}>z_{j}\right), \\
& \boldsymbol{d}_{p q}=\frac{2 \pi \mathrm{i}}{\alpha_{0} \beta} \frac{\left(k^{2} \mathbf{1}-\boldsymbol{k}_{p q} \boldsymbol{k}_{p q}^{T}\right)}{\kappa_{p q}}=\boldsymbol{d}_{p q}\left(\boldsymbol{k}_{p q}\right), \\
& \boldsymbol{k}_{p q}=\left(\boldsymbol{k}_{p q}^{\|}, k_{p q z}\right)=\left(\boldsymbol{k}_{\|}+\boldsymbol{g}_{p q}^{\|}, \boldsymbol{\kappa}_{p q}\right), \\
& \kappa_{p q}=\left(k^{2}-\left|\boldsymbol{k}_{\|}+\boldsymbol{g}_{p q}^{\|}\right|^{2}\right)^{1 / 2} .
\end{aligned}
$$

In the above equations, use has been made of the following definitions. $g_{p q}^{\|}$is a surface reciprocal lattice vector and $\alpha_{0}$ is a normalization unit for the polarizability and has been defined as $4 \pi \varepsilon_{0} a^{3}$. Equations (9) only hold if $\left(z_{i}-z_{j}\right)$ is positive. If $\left(z_{i}-z_{j}\right)$ becomes negative, $\boldsymbol{d}_{p q}$ in (9b) has to be replaced by:

$\underline{\boldsymbol{d}}_{p q}=\boldsymbol{d}_{p q}\left(\underline{\boldsymbol{k}}_{p q}\right)$.
For the two remaining lattice sums one needs Ewald's one-fold integral transform [10], but in a form generalized for arbitrary lattices, the exact derivation of which will be given in a forthcoming article [6]. Litzman has studied the same transform for the case of square lattices [12]. Litzman was also the first to obtain an expression for the transfer tensor of arbitrary lattices using the three-fold integral transform [13]. For intraplanar lattice sums, governing the transfer tensor $f_{i i}$, which corresponds to the interaction between the characteristic dipole and its own plane, mostly the convention is used by other authors that $\boldsymbol{f}_{i i}=\boldsymbol{\alpha}_{0}^{-1} \boldsymbol{c}$. The components of $\boldsymbol{c}$ for the static case have been studied extensively in the past and yield the famous constants of dipole theory for square and hexagonal lattices [14]. Approximate values for $\boldsymbol{c}$ have been obtained by Vlieger [15].

Equations (8) represent a system of $3 N$ complex simultaneous linear equations, $N$ being the total number of planes making up the slab. They can be reorganized to standard (matrix) form as follows:

$\mathbf{M}^{\mathrm{ss}}\left[\boldsymbol{p}_{i}\right]=\left[\boldsymbol{E}_{\mathrm{ext}, i}\right]$,

$\mathbf{M}_{i j}^{\mathrm{ss}}=\boldsymbol{\alpha}_{i}^{-1} \delta_{i j}-\boldsymbol{f}_{i j}$.

The matrix $\mathbf{M}^{\text {ss }}$ will be called the interaction matrix for the slab problem and is composed of $(N \times N)(3 \times 3)$ tensors of type (11b). The quantity $\left[\boldsymbol{p}_{i}\right]$ represents a compound vector containing the $N$ unknown vectors $\boldsymbol{p}_{i} .\left[\boldsymbol{E}_{\text {ext, },}\right]$ has a similar meaning. Upon solution of eqs. (11), the $\boldsymbol{p}_{i}$ control completely the optical response of the slab.

The remote fields emitted by those dipoles (reflected and transmitted) have to be found from the transfer tensor ( $7 b$ ) which is calculated by means of the three-fold integral transform. In that case one arrives at expressions similar to (9). For remote fields only the $(p, q) \neq(0,0)$ term has to be taken into account. Those fields are for reflection resp. transmission;

$$
\begin{aligned}
& \boldsymbol{E}^{\mathrm{r}}(\boldsymbol{r}, t)=\boldsymbol{d}_{00} \mathrm{e}^{\mathrm{i}(\boldsymbol{k} r-\omega t)} \boldsymbol{P}^{\mathrm{r}}, \\
& \boldsymbol{E}^{\mathrm{t}}(\boldsymbol{r}, t)=\boldsymbol{E}_{\mathrm{ext}}(\boldsymbol{r}, t)+\underline{\boldsymbol{d}}_{00} \mathrm{e}^{\mathrm{i}(\boldsymbol{k} r-\omega t)} \boldsymbol{P}^{\mathrm{t}},
\end{aligned}
$$


where the vectors $\boldsymbol{P}^{\mathrm{r}}$ and $\boldsymbol{P}^{\mathrm{t}}$ are defined as:

$\boldsymbol{P}^{\mathbf{r}}=\sum_{i} \mathrm{e}^{-\mathrm{i} \boldsymbol{k} \boldsymbol{r}_{i}} \boldsymbol{p}_{i}$,

$\boldsymbol{P}^{\mathrm{t}}=\sum_{i} \mathrm{e}^{-\mathbf{i} k r_{i}} \boldsymbol{p}_{i}$

Expressions $(12,13)$ display a simple appearance. However, one should not be mislead by this simple behaviour and bear in mind that exactly at this place classical continuum approaches are facing the problem of the boundary conditions. Basically boundary conditions yield also simple descriptions, but that picture changes drastically if phenomena taking place in the few outermost layers of the solid (reconstructions, relaxation, ad- or absorbed monolayers, etc.) have to be incorporated $[2,16,17]$. It is the virtue of the exact solution of the discrete dipole description that the expressions for the remote fields survive such move unaltered. After (13) it is trivial to find the expressions for the reflectivity and transmittivity coefficients of the thin dielectric slab:

$$
\begin{aligned}
& r=\left(\frac{i k^{2}}{2 \varepsilon_{0} \beta k_{z} a^{2}}\right)\left(\frac{\boldsymbol{E}_{0} \cdot \boldsymbol{P}^{\mathrm{r}}}{\left|\boldsymbol{E}_{0}\right|}\right), \\
& t=1+\left(\frac{i k^{2}}{2 \varepsilon_{0} \beta k_{z} a^{2}}\right)\left(\frac{\boldsymbol{E}_{0} \cdot \boldsymbol{P}^{\mathrm{t}}}{\left|\boldsymbol{E}_{0}\right|}\right) .
\end{aligned}
$$

In (14b) it has tacitly been understood that the slab contribution to the transmitted field has the same polarization as the exciting lightbeam, which is true for the cases studied in this article.

\subsubsection{Optical response of the bulk}

The classical continuum descriptions allow for a direct treatment of the electromagnetic wave propagating under bulk circumstances. It suffices to solve the bulk dispersion equation:

$$
\left[\nabla^{2}-\varepsilon \mu_{0} \frac{\partial^{2}}{\partial t^{2}}\right] \boldsymbol{E}(\boldsymbol{r}, t)=0
$$

which follows directly from the macroscopic Maxwell equations and is by no means hard to solve. Combined with the traditional boundary equations, the dispersion equation yields both the Fresnel coefficients and the Snell-Descartes law of refraction. The analogous treatment in the discrete dipole approximation is associated with a normal mode decomposition. Although Ewald made some first steps into that direction at the beginning of this century [10], it has undoubtedly been Litzman and Rózsa [9, 13], who have developed the subject in full mathematical rigour. In a less abstract way and adapted for general cases we will give here their treatment for the bulk normal mode decomposition and the dispersion equation for the discrete case.

At first a bulk unit cell has to be chosen, which apart from obeying the parallel translational symmetry, also is repeated ad infinitum along the positive $z$-axis. Each bulk cell will contain $N_{\text {B }}$ charactcristic dipoles, each controlling its own bulk plane of dipoles, and will have a height of $d_{\mathrm{B}}$. The characteristic dipoles will be located at the sites $\boldsymbol{r}_{v, v}$ given by:

$$
r_{v, V}=r_{v}^{\mathrm{B}}+V d_{\mathrm{B}} \hat{z}
$$

Capital indices refer to bulk cells and small indices to bulk characteristic dipoles. Indices are positive and $r_{1}^{\mathrm{B}}=0$ always. Through (16) we are dealing with a bulk-truncated system since $V$ starts at 0 . Like Litzman we define the normal modes through:

$$
\boldsymbol{p}_{v, V}=\sum_{m=1}^{M} \nu_{m} \boldsymbol{u}_{m v} \mathrm{e}^{\mathrm{i} q_{m^{a V}}}
$$

In principle there will be infinitely many normal modes. The $q_{m}$ correspond to the $z$-component of the 
wave vector of the normal modes, which represent dipole waves (Ewald's "Dipolwellen"). The orientation in real space of the normal modes is controlled by $\boldsymbol{u}_{m v}$ and their strength by $\nu_{m}$. Calculation of the $q_{m}$ and $u_{m v}$ by means of a generalization of Litzman's procedure can be extremely time consuming. The following commensurability theorem can be used to reduce the computational effort drastically for cases where there is only one type of dipoles in the elementary bulk cell. In that case the following relation holds:

$$
\begin{aligned}
& \boldsymbol{u}_{m v}=\mathrm{e}^{i \boldsymbol{k}_{m} \boldsymbol{r}_{v}^{\mathrm{B}}} \boldsymbol{u}_{m}, \\
& \boldsymbol{k}_{\boldsymbol{m}}=\left(k_{x}, k_{y}, q_{m}\right) .
\end{aligned}
$$

We write $\boldsymbol{u}_{m}$ for $\boldsymbol{u}_{m 1}$. The validity of this commensurability theorem will be discussed in a forthcoming article. Use of eqs. (17) and (18) in eqs. (8a, b) and proper replacement of the indices $i, j$ by the sets $(v$, $V),(w, W)$, where $v, w$ run from 1 to $N_{\mathrm{B}}$ and $V, W$ from 0 to infinity, yield the following (infinite) set of simultaneous equations:

$$
\begin{gathered}
\sum_{m=1}^{M} \nu_{m} \sum_{w=1}^{N_{\mathrm{B}}}\left[\sum_{p, q} \frac{\boldsymbol{d}_{p q} \mathrm{e}^{-\mathrm{i} \boldsymbol{k}_{p q} \boldsymbol{r}_{w}^{\mathrm{B}}}}{1-\exp \left(\mathrm{i}\left(q_{m}-\kappa_{p q}\right) d_{\mathrm{B}}\right)}\right] \mathrm{e}^{\mathrm{i} \boldsymbol{k}_{m} \boldsymbol{r}_{w}^{\mathrm{B}}} \mathrm{e}^{\mathrm{i} \boldsymbol{k}_{p q} d_{B} V} \boldsymbol{u}_{m} \\
+\sum_{m=1}^{M} \nu_{m} \mathrm{e}^{\mathrm{i} q_{m} d_{\mathrm{B}} V} \boldsymbol{a}_{m}\left(\boldsymbol{k}_{m}\right) \boldsymbol{u}_{m}=\boldsymbol{E}_{0} \mathrm{e}^{\mathrm{i} \boldsymbol{k} r_{\mathrm{B}}} \mathrm{e}^{\mathrm{i} \boldsymbol{k}_{z} d_{\mathrm{B}} V},
\end{gathered}
$$

where the index $W$ has disappeared as a result of summation and $a\left(k_{m}\right)$ follows from:

$$
\begin{aligned}
\boldsymbol{a}\left(\boldsymbol{k}_{m}\right)= & {\left[\boldsymbol{\alpha}_{B}^{-1}-\alpha_{0}^{-1} \boldsymbol{c}-\sum_{w=2}^{N_{\mathrm{B}}} \mathrm{e}^{\mathrm{i} \boldsymbol{k}_{m} r_{w}^{\mathrm{B}} \boldsymbol{f}_{1, w}^{\mathrm{B}}}\right.} \\
& -\sum_{w=1}^{N_{\mathrm{B}}} \mathrm{e}^{\mathrm{i} k_{m} r_{w}^{\mathrm{B}}}\left[\sum_{p, q}\left(\frac{\boldsymbol{d}_{p q} \mathrm{e}^{-\mathrm{i} \boldsymbol{k}_{p q} \boldsymbol{r}_{w}^{\mathrm{B}}}}{1-\exp \left(\mathrm{i}\left(q_{m}-\kappa_{p q}\right) d_{\mathrm{B}}\right)}+\frac{\underline{\boldsymbol{d}}_{p q} \mathrm{e}^{-\mathrm{i} \underline{k}_{p q} r_{w}^{\mathrm{B}}}}{1-\exp \left(-\mathrm{i}\left(q_{m}+\kappa_{p q}\right) d_{\mathrm{B}}\right)}\right)\right] .
\end{aligned}
$$

$\boldsymbol{\alpha}_{\mathrm{B}}$ represents the bulk polarizability tensor. Further the $\boldsymbol{f}_{1 w}$ are subtensors of the type (8b) but calculated for two planes with characteristic dipoles belonging to the same bulk cell. In that case any explicit $V, W$ dependence disappears. The fact that (19) represents an infinite set of simultaneous equations can also be expressed in the more transparent way that a single equation of type (19) has to hold independent from the value of $V$. Since $q_{m} \neq k_{z}$ one obtains from this requirement, as a first result that:

$$
a\left(k_{m}\right) u_{m}=0
$$

This equation yields only non-trivial solutions if

$$
\operatorname{Det}\left(\boldsymbol{a}\left(\boldsymbol{k}_{m}\right)\right)=0 \text {. }
$$

The equations (20)-(22) are of vital importance for discrete dipole calculations in general. For this reason we will discuss their use and physical interpretation. The $q_{m}$ for the normal modes have to be obtained from (22). Since $a\left(k_{m}\right)$ depends in a non-linear way on the $q_{m}$, the solutions cannot be obtained by means of a simple eigenvalue routine. Only by using non-linear "scanning" routines, solutions can be found. The non-linear dependence is also responsible for the fact that one finds 
infinitely many solutions for (22). Since the $q_{m}$ are the wave vectors for the dipole waves, which control completely the electric fields inside the solid, eq. (22) is the direct equivalent of the Snell-Descartes law in the continuum case. The dispersion relation (15) has no direct equivalent in the discrete dipole theory. The $q_{m}$ obtained for the continuum and discrete approach relate to different physical quantities. In the continuum case the $q_{m}$ describe the macroscopic electric fields being defined everywhere inside the solid. The $q_{m}$ in the discrete case describe dipole waves being defined only at discrete lattice positions. After the $q_{m}$ have been obtained the normal mode vectors $\boldsymbol{u}_{m}$ can be calculated from (21). Since eq. (20) does not contain the index $V$ explicitly, eqs. (21) and (22) are valid everywhere in the bulk. From this one cannot conclude that the $q_{m}, \boldsymbol{u}_{m}$ are pure bulk quantities, since through $\boldsymbol{k}_{\|}$the surface enters (20) in an implicit way. The remaining coefficients $\nu_{m}$ have to be found from (19), which generates a vector equation for each pair $(p, q)$. For optics it suffices to consider the $(0,0)$ equations only, being:

$$
\begin{aligned}
& \sum_{m=1}^{M} \nu_{m} \boldsymbol{g}_{m}=\boldsymbol{E}_{0}, \\
& \boldsymbol{g}_{m}=\frac{\boldsymbol{d}_{00}}{1-\exp \left(\mathrm{i}\left(q_{m}-k_{z}\right) d_{\mathrm{B}}\right)} \sum_{w=1}^{N_{\mathrm{B}}} \mathrm{e}^{\mathrm{i}\left(q_{m}-k_{z}\right) z_{w}^{\mathrm{B}}} \boldsymbol{u}_{m} .
\end{aligned}
$$

This result is just another mathematical formulation of the Ewald-Oseen extinction theorem. It will be discussed elsewhere that in order to calculate the optical response of the bulk only the two normal modes being quasi-nonevanescent (i.e. having a decay length larger than the width of at least 10 monolayers) need to be considered [6]. The other normal modes affect only the surface region, being close to $V=0$. After $M$ has been restricted to 2, (23) has become a dependent set of equations. This dependency can be resolved in an optimal way through projection. Define the projection vectors $\boldsymbol{g}_{S}, \boldsymbol{g}_{P}$ as follows:

$$
\begin{aligned}
& \boldsymbol{g}_{S}=\boldsymbol{d}_{00} \cdot \boldsymbol{u}_{1}, \\
& \boldsymbol{g}_{P}=\boldsymbol{d}_{00} \cdot \boldsymbol{u}_{2} .
\end{aligned}
$$

Now we project the equations onto those vectors, yielding:

$$
\| \begin{array}{ll}
-\left(\boldsymbol{g}_{S} \cdot \boldsymbol{g}_{1}\right) & -\left(\boldsymbol{g}_{S} \cdot \boldsymbol{g}_{2}\right) \\
-\left(\boldsymbol{g}_{P} \cdot \boldsymbol{g}_{1}\right) & -\left(\boldsymbol{g}_{P} \cdot \boldsymbol{g}_{2}\right)
\end{array}||\left|\begin{array}{l}
\nu_{1} \\
\nu_{2}
\end{array}\right|=\left|\begin{array}{l}
\boldsymbol{g}_{S} \cdot \boldsymbol{E}_{0} \\
\boldsymbol{g}_{P} \cdot \boldsymbol{E}_{0}
\end{array}\right|
$$

In appendix $\mathrm{A}$ it will be shown that the off-diagonal elements of (25) are zero. The bulk interaction equations can be written concisely as:

$$
\mathbf{M}^{B B}\left[\nu_{1}\right]=\left[\boldsymbol{g}_{S, P} \boldsymbol{E}_{0}\right]
$$

We have remarked already that the set (25) is not really bulklike in the same sense as the dispersion equation (15). Assuming that the quasi-evanescent modes are the only ones present also at the surface, it is possible to calculate a reflection coefficient from (14a) by replacing (13a) by:

$$
\boldsymbol{P}^{\mathrm{I}}=\sum_{m=1}^{2}\left[\sum_{w=1}^{N_{\mathrm{B}}} \frac{\mathrm{e}^{\mathrm{i}\left(q_{m}+k_{z}\right) z_{\mathrm{B} w}}}{1-\exp \left(\mathrm{i}\left(q_{m}+k_{z}\right) d_{\mathrm{B}}\right)}\right] \nu_{m} \boldsymbol{u}_{m}
$$


A transmission coefficient obviously has no physical meaning in this case. Litzman [9] has shown that (27) reproduces exactly the results obtained from the Fresnel formulas for the case of small $k$, cubic crystals and perpendicular incidence. In all other cases deviations can and have been found, deviations which increase if the surface region has been treated properly (see further in this article). One of the most charming aspects of the discrete approach is the absolute disappearance of boundary conditions. However, neither of the two approaches we presented in this section is able to describe a realistic surface configuration properly. Solely a hybrid method as the double cell method developed by the authors [5,6] (or the comparable, but less efficient two slab method [8]), is capable of handling this problem properly. Especially the double cell method is very computer efficient; computer time is decreased by a factor of at least 16 and the memory requirements by more than 4 compared to the two slab method. In the following section an extension of this method to the case of thick slabs is described.

\subsection{Optical reponse of thick slabs}

The thick slab method is a further development of the double cell method. Both methods use the idea of merging the approaches of sections 2.3.1 (thin slabs/real space) and 2.3.2 (bulk/normal mode decomposition). The link will be established by means of a projection technique. The entire thick slab is going to be composed of three subslabs, the first and third being described in real space, the second thick intermediate slab by means of normal modes. The thick slab is located in the positive upper halfspace $(z \geqslant 0)$. The first subslab (which will be labelled I) contains $N_{\mathrm{S}}^{\mathrm{I}}$ dipole layers and has thickness $d_{\mathrm{S}}$. The bulklike intermediate subslab contains $N$ bulk cells and its characteristic dipoles are located at:

$$
r_{v, V}=d_{\mathrm{s}} \hat{z}+r_{v}^{\mathrm{B}}+V d_{\mathrm{B}} \hat{z}
$$

The meaning of concepts and symbols is like in previous sections. The third subslab (labelled II) resembles the first and continues after the second. It contains $N_{\mathrm{s}}^{\mathrm{II}}$ dipole layers. Care has been taken that the bulk configuration as given by (28) extends in the neighbouring surface subslabs. The fact that the boundaries between the subslabs are by construction inside of the bulklike area, is vital for the approach. An arbitrary characteristic dipole $i$, located in the first surface subslab, has to obey:

$$
\begin{aligned}
& \left(\boldsymbol{\alpha}_{s}^{-1}-\alpha_{0}^{-1} \boldsymbol{c}\right) \boldsymbol{p}_{1}-\sum_{j=1}^{N{ }_{S}^{\prime}} \boldsymbol{f}_{i j} \boldsymbol{p}_{j}-\sum_{m=1}^{M} \underline{g}_{m}^{i} \nu_{m}-\sum_{j=N_{\mathrm{S}}^{\mathrm{I}}+1}^{N_{\mathrm{S}}} \boldsymbol{f}_{i j} \boldsymbol{p}_{j}=\boldsymbol{E}_{i}, \\
& \underline{\boldsymbol{g}}_{m}^{i}=\sum_{v=1}^{N_{\mathrm{B}}} \sum_{p, q} \underline{\boldsymbol{d}}_{p q} \mathrm{e}^{\mathrm{i} \underline{\boldsymbol{k}}_{p q}\left(\boldsymbol{r}_{i}-\boldsymbol{r}_{\mathrm{B} v}\right)} \mathrm{e}^{\mathrm{i} \kappa_{p q} d_{\mathrm{S}}}\left(\frac{1-\mathrm{e}^{\mathrm{i}\left(q_{m}+\kappa_{p q}\right) d_{\mathrm{B}} N}}{1-\mathrm{e}^{\mathrm{i}\left(q_{m}+\kappa_{p q}\right) d_{\mathrm{B}}}}\right) \mathrm{e}^{\mathrm{i} \boldsymbol{k}_{m} \boldsymbol{r}_{\mathrm{B} v}} \boldsymbol{u}_{m} .
\end{aligned}
$$

The index for the second surface subslab continues uninterrupted from the last index of the first. $N_{\mathrm{S}}$ is the total number of dipole layers in the two surface subslabs: $N_{\mathrm{S}}=N_{\mathrm{S}}^{\mathrm{I}}+N_{\mathrm{S}}^{\mathrm{II}} \cdot N_{\mathrm{B}}$ indicates the number of dipole layers in a bulk unit cell, exactly as in eq. (19). If the dipole $i$ happens to be located in the second surface subslab an expression completely analogous to (29) will be obtained (in that case the prime moves to the second surface summation and all underscores, i.e. for $\underline{\boldsymbol{g}}_{m}^{i}, \underline{\boldsymbol{k}}_{p q}$ and $\underline{\boldsymbol{d}}_{p q}$ disappear).

The description of the bulklike intermediatc slab follows closcly the lines of thought represented in section 2.3.2. In particular the expression for $a\left(k_{m}\right)$ as given by eq. (20) remains unaltered. Hence we obtain also the same sets of corresponding eigenvalues $q_{m}$ and eigenvectors $\boldsymbol{u}_{m}$, as found from (21) and (22). The real differences concern eq. (23), the extinction equation. In the bulk we had to worry only about dipole waves moving in upward direction, since the downward solutions lead to unphysical results. This restriction no longer holds for slabs and hence we have to include also the two 
quasi-nonevanescent modes propagating in the negative $z$-direction. This brings the number of required normal modes at $M=4$.

Since we assume that there is no optical activity (the theory however can easily be extended for those cases, since optical activity requires only a change in the $\boldsymbol{\alpha}_{\imath}$ ), the two extra normal mode wave vectors have to obey:

$$
q_{3}=-q_{1}, \quad q_{4}=-q_{2}
$$

and the corresponding normal mode eigenvectors:

$$
\boldsymbol{u}_{3}=\underline{u}_{1}, \quad \boldsymbol{u}_{4}=\underline{u}_{2}
$$

Instead of one vector equation, governing the bulk behaviour, like eq. (23), we obtain for the bulk of a thick slab two vector equations, being:

$$
\begin{aligned}
& -\sum_{j=1}^{N_{\mathrm{S}}^{1}} \boldsymbol{d}_{00} \mathrm{e}^{-\mathrm{i} \boldsymbol{k} r_{j}} \boldsymbol{p}_{j}-\sum_{m=1}^{4} \nu_{m} \boldsymbol{g}_{m}=\boldsymbol{E}_{0}, \\
& -\sum_{j=N_{\mathrm{S}}^{1}+1}^{N_{\mathrm{S}}} \underline{\boldsymbol{d}}_{00} \mathrm{e}^{-\mathrm{i} \boldsymbol{k} r_{j}} \boldsymbol{p}_{j}+\sum_{m=1}^{4} \nu_{m} \underline{\boldsymbol{g}}_{m}=0,
\end{aligned}
$$

with

$$
\begin{aligned}
& \boldsymbol{g}_{m}=\sum_{w=1}^{N_{\mathrm{B}}} \boldsymbol{d}_{00} \mathrm{e}^{-\mathrm{i} k_{z} d_{\mathrm{S}}}\left(\frac{\mathrm{e}^{-\mathrm{i}\left(k_{z}-q_{m}\right) z_{w}^{\mathrm{B}}}}{1-\mathrm{e}^{-\mathrm{i}\left(k_{z}-q_{m}\right) d_{\mathrm{B}}}}\right) \boldsymbol{u}_{m}, \\
& \underline{\boldsymbol{g}}_{m}=\sum_{w=1}^{N_{\mathrm{B}}} \underline{\boldsymbol{d}}_{00} \mathrm{e}^{\mathrm{i} k_{z} d_{\mathrm{S}}}\left(\frac{\mathrm{e}^{\mathrm{i}\left(k_{z}+q_{m}\right)\left(z_{w}^{\mathrm{B}}+N d_{\mathrm{B}}\right)}}{1-\mathrm{e}^{\mathrm{i}\left(k_{z}+q_{m}\right) d_{\mathrm{B}}}}\right) \boldsymbol{u}_{m} .
\end{aligned}
$$

Again the dependence has to be removed by means of projection. For this eqs. (24) have to be extended:

$$
\underline{\boldsymbol{g}}_{S}=\underline{\boldsymbol{d}}_{00} \boldsymbol{u}_{3}, \quad \underline{\boldsymbol{g}}_{P}=\underline{\boldsymbol{d}}_{00} \boldsymbol{u}_{4} .
$$

We arrive after projection at a set of 4 complex equations, which can be written in an explicit way like

$$
\begin{aligned}
& -\sum_{j=1}^{N_{S}^{\mathrm{I}}}\left(\boldsymbol{g}_{S} \boldsymbol{d}_{00}\right) \mathrm{e}^{-\mathrm{i} k r_{j}} \boldsymbol{p}_{j}-\nu_{1}\left(\boldsymbol{g}_{S} \boldsymbol{g}_{1}\right)-\nu_{3}\left(\boldsymbol{g}_{S} \boldsymbol{g}_{3}\right)=\boldsymbol{g}_{S} \boldsymbol{E}_{0}, \\
& -\sum_{j=1}^{N_{S}^{\mathrm{I}}}\left(\boldsymbol{g}_{P} \boldsymbol{d}_{00}\right) \mathrm{e}^{-\mathrm{i} \boldsymbol{k} r_{j}} \boldsymbol{p}_{j}-\nu_{2}\left(\boldsymbol{g}_{P} \boldsymbol{g}_{2}\right)-\nu_{4}\left(\boldsymbol{g}_{P} \boldsymbol{g}_{4}\right)=\boldsymbol{g}_{S} \boldsymbol{E}_{0}, \\
& \nu_{1}\left(\underline{\boldsymbol{g}}_{S} \underline{\boldsymbol{g}}_{1}\right)+\nu_{3}\left(\underline{\boldsymbol{g}}_{S} \underline{\boldsymbol{g}}_{3}\right)-\sum_{j=N_{\mathrm{S}}^{\mathrm{I}}+1}^{N_{\mathrm{S}}}\left(\underline{\boldsymbol{g}}_{S} \underline{\boldsymbol{d}}_{00}\right) \mathrm{e}^{-\mathrm{i} k r_{j}} \boldsymbol{p}_{j}=0, \\
& \nu_{2}\left(\underline{\boldsymbol{g}}_{P} \underline{\boldsymbol{g}}_{2}\right)+\nu_{4}\left(\underline{\boldsymbol{g}}_{P} \underline{\boldsymbol{g}}_{4}\right)-\sum_{j=N_{\mathrm{S}}^{\mathrm{I}}+1}^{N_{\mathrm{S}}}\left(\underline{\boldsymbol{g}}_{P} \underline{\boldsymbol{d}}_{00}\right) \mathrm{e}^{-\mathrm{i} \boldsymbol{k} r_{j}} \boldsymbol{p}_{j}=0 .
\end{aligned}
$$


Here, again use has been made of the results of appendix A. Results (31) and (34) can be written in matrix form and the corresponding interaction equations are given by (35):

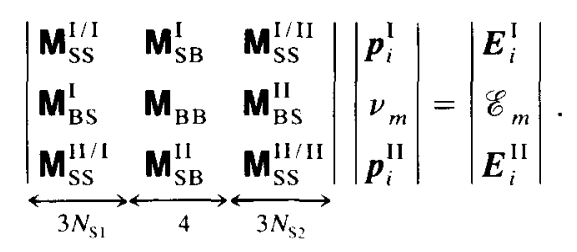

The total interaction matrix consists of 9 submatrices and the meaning of all components of (35) follows clearly after comparison with eqs. (29) and (34). We will write down more explicitly some elements of the middle (bulk) row:

$$
\begin{aligned}
\mathbf{M}_{\mathrm{BS}, m j}^{\mathrm{I}} & =-\mathrm{e}^{\mathrm{i} \boldsymbol{k r} r_{j}}\left(\boldsymbol{g}_{S} \boldsymbol{d}_{00}\right) & & (m=1,2), \\
& =0 & & (m=3,4) .
\end{aligned}
$$

The central part of the bulk row $\mathbf{M}_{\mathrm{BB}}$ becomes:

$$
\mathbf{M}_{\mathrm{BB}}=\left|\begin{array}{cccc}
-\left(\boldsymbol{g}_{S} \boldsymbol{g}_{1}\right) & 0 & -\left(\boldsymbol{g}_{S} \boldsymbol{g}_{3}\right) & 0 \\
0 & -\left(\boldsymbol{g}_{P} \boldsymbol{g}_{2}\right) & 0 & -\left(\boldsymbol{g}_{P} \boldsymbol{g}_{4}\right) \\
\left(\underline{g}_{S} \boldsymbol{g}_{1}\right) & 0 & \left(\underline{\boldsymbol{g}}_{S} \boldsymbol{g}_{3}\right) & 0 \\
0 & \left(\underline{\boldsymbol{g}}_{P} \boldsymbol{g}_{2}\right) & 0 & \left(\underline{\boldsymbol{g}}_{P} \boldsymbol{g}_{4}\right)
\end{array}\right|
$$

Finally, we have to specify the bulklike inhomogeneous vector used in (35):

$$
\left|\begin{array}{l}
\mathscr{E}_{1} \\
\mathscr{E}_{2} \\
\mathscr{E}_{3} \\
\mathscr{E}_{4}
\end{array}\right|=\left|\begin{array}{l}
\left(g_{s} \boldsymbol{E}_{0}\right) \\
\left(\boldsymbol{g}_{P} \boldsymbol{E}_{0}\right) \\
0 \\
0
\end{array}\right|
$$

The solution of the set of interaction eqs. (35) yields the unkown $\boldsymbol{p}_{i}^{\mathbf{I}}, v_{m}$ and $\boldsymbol{p}_{i}^{\mathrm{II}}$, being resp. $N_{\mathrm{s}}^{\mathrm{I}}$ vectors, 4 complex numbers and $N_{\mathrm{s}}^{\mathrm{II}}$. From these unknown variables immediately follow the reflection and transmission coefficients for the thick slab. One can use eqs. (14) directly, provided one makes use of the following expressions of $\boldsymbol{P}^{\mathrm{r}}$ and $\boldsymbol{P}^{\mathrm{t}}$ :

$$
\begin{aligned}
\boldsymbol{P}^{\mathrm{r}} & =\sum_{j=1}^{N_{\mathrm{S}}} \mathrm{e}^{-\mathrm{i} \underline{k} r_{j}} \boldsymbol{p}_{j}+\mathrm{e}^{\mathrm{i} k_{z} d_{\mathrm{S}}} \sum_{m=1}^{4}\left[\sum_{w=1}^{N_{\mathrm{B}}} \mathrm{e}^{\mathrm{i}\left(k_{z}+q_{m}\right) z_{w}^{\mathrm{B}}}\left(\frac{1-\mathrm{e}^{\mathrm{i}\left(k_{z}+q_{m}\right) N d_{\mathrm{B}}}}{1-\mathrm{e}^{\mathrm{i}\left(k_{z}+q_{m}\right) d_{\mathrm{B}}}}\right)\right] \nu_{m} \boldsymbol{u}_{m}, \\
\boldsymbol{P}^{\mathbf{t}} & =\sum_{j=1}^{N_{\mathrm{S}}} \mathrm{e}^{-\mathrm{i} \boldsymbol{k} r_{j}} \boldsymbol{p}_{j}+\mathrm{e}^{-\mathrm{i} k_{z} d_{\mathrm{S}}} \sum_{m-1}^{4}\left[\sum_{w-1}^{N_{\mathrm{B}}} \mathrm{e}^{-\mathrm{i}\left(k_{z}-q_{m}\right) z_{w}^{\mathrm{B}}}\left(\frac{1-\mathrm{e}^{-\mathrm{i}\left(k_{z}-q_{m}\right) N d_{\mathrm{B}}}}{1-\mathrm{e}^{-\mathrm{i}\left(k_{z}-q_{m}\right) d_{\mathrm{B}}}}\right)\right] \nu_{m} \boldsymbol{u}_{m} .
\end{aligned}
$$

The summation over the two surface layers has been combined in this expression.

\section{Calculation of a few model configurations}

In order to illustrate the impact of the theoretical model, treated in the previous sec- tion, we will show now the results of some actual calculations displaying the characteristic features.

Basically the entire thick slab has been taken 
as a part of a simple cubic lattice with lattice parameter $a$. So all coordinates of the slab obey:

$r_{n m l}=n a \hat{x}+m a \hat{y}+l a \hat{z}$.

As a result the characteristic dipoles for all planes making up the slab (the $r_{i}$ in eq. (4)) will be located along the $z$-axis and it is very easy to obtain the specific choices of coordinates for each of the subslabs. Each site $i$ will be given a polarizability tensor $\boldsymbol{\alpha}_{i}$ being the samc for all sites. This common $\boldsymbol{\alpha}$ will be supposed to be diagonal and isotropic. Consequently a simple complex number $\alpha$ suffices to describe the optical properties of the slab. Under those presumptions the entire character of the solutions will be determined by a single parameter $\alpha / \alpha_{0}$. This parameter roughly distinguishes the ratio in which internal and external fields contribute to the local field. Therefore we have chosen to focus upon the following three cases:

(a) weak coupling case (WCC): $\alpha / \alpha_{0}=0.05+\mathrm{i} 0.0$,

(b) strong coupling case (SCC): $\alpha / \alpha_{0}=0.2+\mathrm{i} 0.0$,

(c) strong coupling/absorbing case (SAC): $\alpha / \alpha_{0}=0.2+\mathrm{i} 0.005$.

Weak and strong coupling specifies the amount in which a specific dipole becomes influenced by its environment.

Primarily the situation occurring for perpendicularly incident light will be considered. So we will not specify the angle of incidence and for the few cases which will be discussed at the end where angular dependency will be investigated, this will be mentioned explicitly. First we consider a simple thin slab, for which results can be obtained using the approach of section 2.3.1. We show in fig. 1 the absolute value of the induced dipole strengths for a slab consisting of 17 layers and a frequency of $5.0 \mathrm{eV}$. In order to make comparison easier, results have been normalized with respect to an average dipole strength $P_{\mathrm{av}}$ defined as:
$P_{\mathrm{av}}=\sum_{i=1}^{N_{\mathrm{S}}}\left|\boldsymbol{p}_{i}\right| / N_{\mathrm{S}}$,

where $N_{\mathrm{s}}$ represents the number of dipoles in the thin slab this time. For weak coupling the dipole strength's length is almost constant in the slab. Only for the first and perhaps also for the second outermost layers, slight deviations with respect to the interior values can be observed. The two strong coupling cases, however, display a more peculiar behaviour. The normalized absolute values of the dipole strengths are far from constant now. For the 5 outermost layers a rapid oscillatory behaviour can be observed $[18,19]$ to which further on will be referred to as 'wiggles'. Figure 1 shows also another unexpected feature. Although the light beam passes through the sample going from left to right, we see, quite against expectation, that the dipole strengths increase in the direction of the light in the interior region (from $i=5$ to 13) of the slab, but it is a correct result. The wiggles near the surface are intriguing and we have further investigated this phenomenon in fig. 2. Here we have calculated the same property as in fig. 1 , but with the thick slab method, for the case of absorbing strong coupling. We want to investigate if the wiggles affect the convergence behaviour. Figure 2 shows four curves, one of them being the result shown

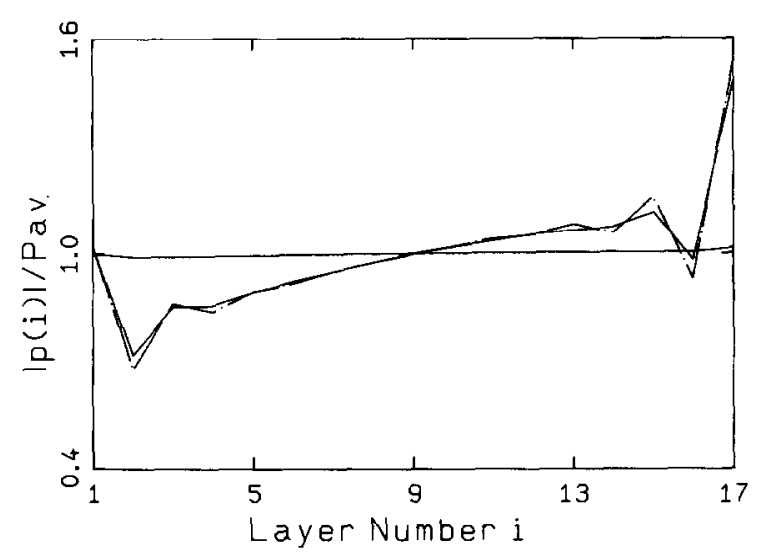

Fig. 1. Absolute values of the dipole strength $p_{i}=\left|p_{i}\right|$, normalized with respect to $P_{\mathrm{av}}$, plotted as a function of the layer index $i$ for a slab of 17 layers. Photon energy $5.0 \mathrm{eV}$. Angle of incidence $\theta_{i}=0$. Almost straight line; WCC, solid line; SAC, chain dotted line; SCC. 


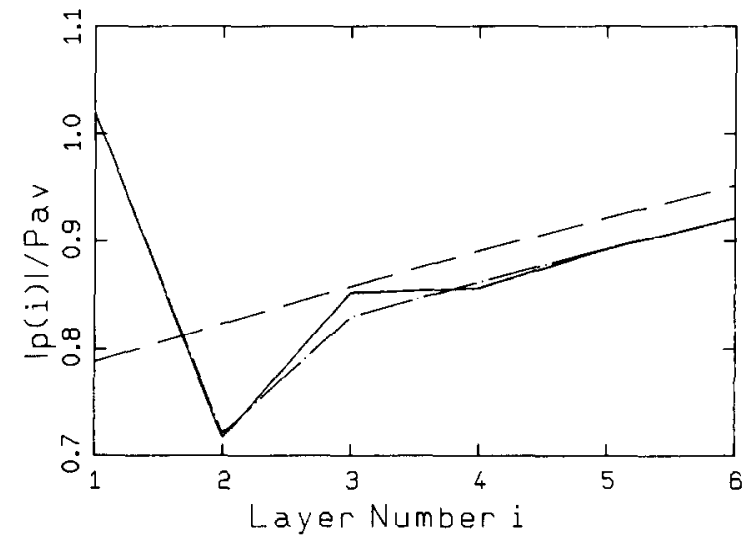

Fig. 2. Convergence behaviour of $p_{i}$, SAC-case. Energy $5.0 \mathrm{eV}, \theta_{i}=0$. Dashed line; $N_{\mathrm{s} 1}=0$, chain-dotted line; $N_{\mathrm{s} 1}=$ 2 , solid curve; coinciding $N_{\mathrm{s} 1}=4$ and thin slab results.

already in fig. 1. This is the limiting case, where all the dipole strengths are calculated in a real space approach. Only the first surface slab is shown. The other curves show how the results vary if the number of layers in the surface slab increases from 0 and 2 to 4 . The latter result is virtually indistinguishable from the thin slab calculation. Hence we can conclude that after already 5 layers in the surface slab full convergence has been obtained. Since the wiggles also extend over 5 layers, the suggested link seems to be plausible. Further confirmation of this hypothesis is obtained if we concentrate upon reflectivity and transmittivity of the slabs. From the thin slab calculation which serves as a reference we obtain the following results for a 17 layer slab assuming case (c) (SAC):

$$
\begin{aligned}
& r=-7.37724 \times 10^{-1}+\mathrm{i} \cdot 2.52060 \times 10^{-1}, \\
& t=3.25186 \times 10^{-1}+\mathrm{i} \cdot 4.10172 \times 10^{-1} .
\end{aligned}
$$

The same results have been obtained by means of the thick slab method and those results are shown in table $1 . N_{\mathrm{S}}$ has the meaning originally defined for eq. (29), i.e. the total number of dipole layers in both surface subslabs (for practical reasons there will be one dipole more in the second surface subslab than in the first). If we limit ourselves to an accuracy of 3 decimal places, we learn from table 1 that for $N_{\mathrm{s}}=10$ full convergence has been achieved. Also this can be rescheduled to a 5 layer thick electromagnetic surface layer. The same result will be obtained for case (b), but for case (a) a surface slab of 2 layers is already enough to obtain convergence. Anyhow, it becomes clear from these calculations that the thick slab method has a very fast convergence rate.

In fig. 3 we continue the inquiry about the local aspects of this discrete dipole approach. The absorption of the incoming energy by the slab is an important physical quantity. It will be

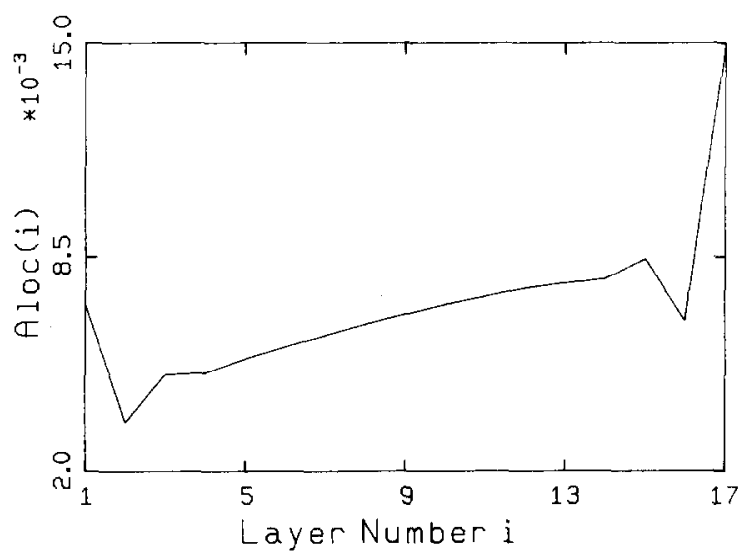

Fig. 3. Local absorbance as a function of the layer index $i$, SAC-case, $\hbar \omega=5.0 \mathrm{eV}, \theta_{i}=0$.

Table 1

\begin{tabular}{rll}
\hline$N_{\mathrm{s}}$ & $r$ & $t$ \\
\hline 0 & $-7.30749 \times 10^{-1}+\mathrm{i} \cdot 2.59416 \times 10^{-1}$ & $3.34102 \times 10^{-1}+\mathrm{i} \cdot 4.14686 \times 10^{-1}$ \\
3 & $-7.38392 \times 10^{-1}+\mathrm{i} \cdot 2.52378 \times 10^{-1}$ & $3.24507 \times 10^{-1}+\mathrm{i} \cdot 4.10098 \times 10^{-1}$ \\
5 & $-7.37469 \times 10^{-1}+\mathrm{i} \cdot 2.52094 \times 10^{-1}$ & $3.25405 \times 10^{-1}+\mathrm{i} \cdot 4.10341 \times 10^{-1}$ \\
7 & $-7.37817 \times 10^{-1}+\mathrm{i} \cdot 2.52017 \times 10^{-1}$ & $3.25117 \times 10^{-1}+\mathrm{i} \cdot 4.10099 \times 10^{-1}$ \\
9 & $-7.37702 \times 10^{-1}+\mathrm{i} \cdot 2.52086 \times 10^{-1}$ & $3.25201 \times 10^{-1}+\mathrm{i} \cdot 4.10204 \times 10^{-1}$ \\
11 & $-7.37728 \times 10^{-1}+\mathrm{i} \cdot 2.52047 \times 10^{-1}$ & $3.25183 \times 10^{-1}+\mathrm{i} \cdot 4.10159 \times 10^{-1}$ \\
13 & $-7.37725 \times 10^{-1}+\mathrm{i} \cdot 2.52064 \times 10^{-1}$ & $3.25185 \times 10^{-1}+\mathrm{i} \cdot 4.10177 \times 10^{-1}$ \\
15 & $-7.37724 \times 10^{-1}+\mathrm{i} \cdot 2.52060 \times 10^{-1}$ & $3.25186 \times 10^{-1}+\mathrm{i} \cdot 4.10171 \times 10^{-1}$ \\
\hline
\end{tabular}


shown [6] that the absorbance of light energy in a separate, say the $i$ th, layer of a slab follows from the expression:

$A_{\mathrm{loc}, i}=-\frac{k^{2}}{\beta k_{z} \varepsilon_{0} a^{2}\left|\boldsymbol{E}_{0}\right|^{2}} \operatorname{Im}\left(\boldsymbol{p}_{i}^{* \mathrm{t}}\left(\boldsymbol{\alpha}_{i}\right)^{-1} \boldsymbol{p}_{i}\right)$,

where the ${ }^{*}$ denotes that the complex conjugate is to be taken and Im stands for "the imaginary part of". From (43) it becomes clear that it is only worthwile to study the local absorbances for case (c). The total absorbance of the slab can be calculated through:

$A=\sum_{i=1}^{N_{\mathrm{TOT}}} A_{\mathrm{loc}, i}$,

where $N_{\text {TOT }}$ means the total number of dipole layers in the slab. The same result, however, can also be obtained from the macroscopic parameters $r$ and $t$ by means of Poynting's theorem. One obtains:

$A=1-\left(r^{*} r\right)-\left(t^{*} t\right)$.

Obviously the microscopic expression (44a) and the macroscopic one (44b) should yield the same result. For the 17 layer slab one obtains agreement between the two results to the last digit, the result being $A=1.1824177 \times 10^{-1}$. The shape of the local absorbance curve shown in fig. 3 highly resembles fig. 1 but only the wiggles have become more pronounced. This is not strange in view of (43), since only a squarc operation lies in between the two figures.

The fact that the backside of a thin slab responds stronger to the incident light than the front has been investigated further in figs. 4 and 5. Both figures relate to the (c)-case (SAC). This time we exploit fully the potential of the thick slab method to calculate exactly slabs up to and including macroscopic sizes, within a rigorous microscopic description. In this way slabs have been calculated containing totally 209 (fig. 4) and 509 (fig. 5) dipole layers. The total number of surface layers has been kept constant at the value 13. From the discussion of fig. 2 it is clear that this value guarantees convergence sufficient-

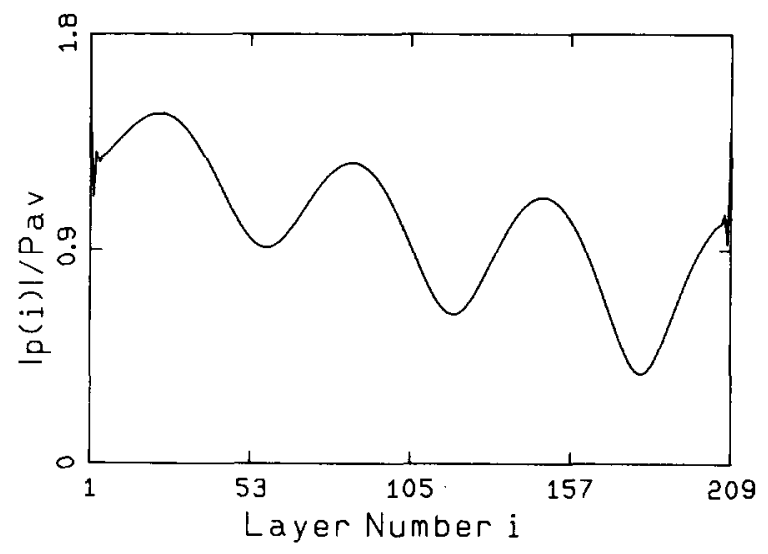

Fig. 4. Normalized absolute values $p_{i} / F_{\text {av }}$ as a function of the layer index $i$ for a slab of 209 layers. SAC-case, $\hbar \omega=5.0 \mathrm{eV}$, $\theta_{i}=0$.

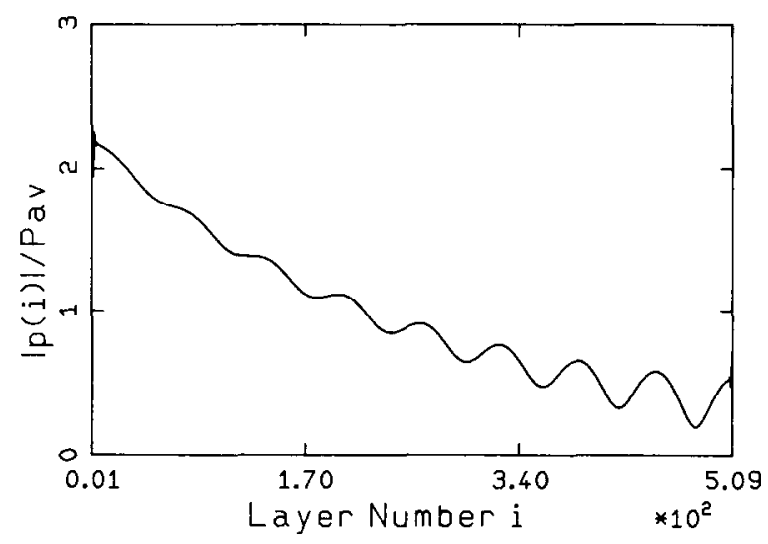

Fig. 5. Same as fig. 4 for a slab of 509 layers.

ly. The absolute values of the dipole strengths shown in figs. 4 and 5 have been normalized again, using this time for $P_{\mathrm{av}}$ :

$P_{\mathrm{av}}=\left(\sum_{i=1}^{N_{\mathrm{S}}}\left|\boldsymbol{p}_{i}\right|+\sum_{V=1}^{N_{1}} \sum_{v=1}^{N_{b}}\left|\boldsymbol{p}_{v, V}\right|\right) /\left(N_{\mathrm{S}}+N \cdot N_{\mathrm{B}}\right)$.

Figure 4 shows an oscillatory behaviour for $\left|\boldsymbol{p}_{i}\right|$ with a period considerably larger than those of the wiggles. Hence, these bulk oscillations have to be interpreted as a simple standing wave pattern caused by back- and forth-scattered bulklike dipole waves of lattice vector $q_{m}$. So this phenomenon has nothing to do with the wiggles, 
which reflect an independent feature of the calculation which is also clearly visible in fig. 4 . 'The bulk oscillations show an increasing strength for the first 25 dipole laycrs from the left (=frontside). It is exactly the onset of this quarter wavelength which we observe in fig. 1 . Hence the increasing dipole strength for very thin slabs has to be interpreted as a macroscopic interference phenomenon. This interpretation is further strengthened by fig. 5 . The largest modulation in fig. 5 can be observed at the right (-backside) of the slab. At the frontside the backward running normal modes have lost their strength so much due to absorption, that interference has almost vanished. If we neglect in fig. 5 the long range oscillations and concentrate upon the overall effect, we see a smoothly decaying dipole wave, most in agreement with our direct intuition. Note also that in fig. 5 the wiggles, although less pronounced due to the change of scale, still are there. It is further also noteworthy that for thin slabs at optical frequencies the strongest local fields occur at the backside of the slab.

The traditional way to calculate the transmission/reflection for thick slabs is by means of the Fresnel formulas. For those formulas one needs a dielectric constant which can be obtained from $\alpha$ by means of the Clausius-Mossotti equation. The reflectivity $r$ and transmittivity $t$ for a semiinfinite system follow from (perpendicular incidence):

$$
\begin{aligned}
& r=(1-\sqrt{\varepsilon}) /(1+\sqrt{\varepsilon}), \\
& t=2 /(1-\sqrt{\varepsilon}) .
\end{aligned}
$$

Those semi-infinite results have to be used in the classical equations for the optical response of slabs as can be found in ref. [20]. They yield the expressions for reflectivity and transmittivity which can be compared with results obtained from (14). Table 2 shows slab reflectances obtained from the thick slab method and obtained with Fresnel, both correponding to 509 dipole layers. The same results have also been calculated for semi-infinite systems. Three parameters have been varied: photon-energy, coupling strength and thickness of the surface slabs.

The most striking conclusion from table 2 is that agreement between classical Fresnel and discrete dipole calculations is at best if the surface slabs are neglected. This means that Fresnel-type calculations neglect surface local field effects. This is also in agreement with the rest of the conclusions. If we increase the coupling rate by going from case (a) to case (c) the difference with respect to Fresnel increases. Since the influence of local fields is determined by the coupling rate, this confirms our earlier conclusions. In general the discrepancy also increases if we increase the energy. This is less easy understood, but it seems that the higher the ratio between the thickness of surface slab and wavelength, the more prominent the surface effects become. In general also slabs show larger deviations than semi-infinite systems. For weak coupling there is even no difference between the two types of calculation for the semi-infinite system.

In fig. 6 the total absorbance for a thick slab of

\begin{tabular}{|c|c|c|c|c|}
\hline \multirow[t]{2}{*}{$\mathbf{R}$} & \multirow[t]{2}{*}{ Energy } & \multirow[t]{2}{*}{ Fresnel } & \multicolumn{2}{|c|}{ Discrete dipole model } \\
\hline & & & $N_{\mathrm{s}}=0$ & $N_{\mathrm{s}}=13$ \\
\hline \multicolumn{5}{|c|}{509 Layers } \\
\hline \multirow[t]{2}{*}{ WCC } & $0.1 \mathrm{eV}$ & $2.594006 \times 10^{-3}$ & $2.594006 \times 10^{-3}$ & $2.594217 \times 10^{-3}$ \\
\hline & $5.0 \mathrm{eV}$ & $4.215521 \times 10^{-2}$ & $4.215971 \times 10^{-2}$ & $4.213094 \times 10^{-2}$ \\
\hline \multirow[t]{2}{*}{$\mathrm{SAC}$} & $0.1 \mathrm{eV}$ & $4.122755 \times 10^{-1}$ & $4.122755 \times 10^{-1}$ & $4.126034 \times 10^{-1}$ \\
\hline & $5.0 \mathrm{eV}$ & $3.671224 \times 10^{-1}$ & $3.672563 \times 10^{-1}$ & $3.689785 \times 10^{-1}$ \\
\hline \multicolumn{5}{|c|}{ Semi-infinite crystal } \\
\hline \multirow[t]{2}{*}{ WCC } & $0.1 \mathrm{eV}$ & $2.107921 \times 10^{-2}$ & $2.107921 \times 10^{-2}$ & $2.107921 \times 10^{-2}$ \\
\hline & $5.0 \mathrm{eV}$ & $2.107921 \times 10^{-2}$ & $2.108021 \times 10^{-2}$ & $2.108021 \times 10^{-2}$ \\
\hline \multirow[t]{2}{*}{ SAC } & $0.1 \mathrm{eV}$ & $3.653626 \times 10^{-1}$ & $3.653626 \times 10^{-1}$ & $3.653864 \times 10^{-1}$ \\
\hline & $5.0 \mathrm{eV}$ & $3.653626 \times 10^{-1}$ & $3.654678 \times 10^{-1}$ & $3.666630 \times 10^{-1}$ \\
\hline
\end{tabular}

Table 2 


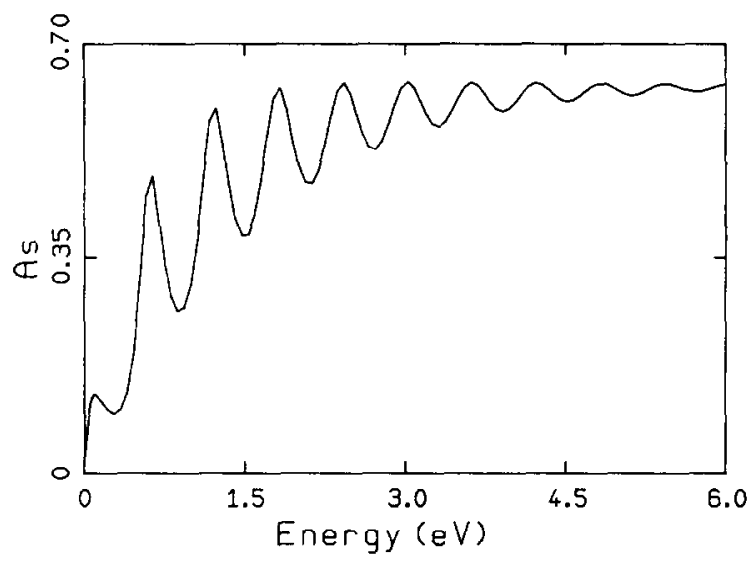

Fig. 6. Total absorbance $A$ of a slab of 509 layers as a function of the photon energy. SAC-case, $\theta_{i}=0$.

509 layers and $N_{\mathrm{S}}=13$ is shown as a function of frequency (case (c)). For low frequencies this absorbance shows strong modulation which for higher frequencies damps out substantially. This has to be understood in the following way. The effective damping of the light beam follows from the imaginary component of $q_{m}$. This turns out to be proportional to the frequency $\omega$. One arrives at similar conclusions if one starts from eq. (43). Hence the disappearance of the modulation has to be attributed to the vanishing of the returning normal mode. The flattening of the curve has a different explanation: the dipole waves have become extinct before they reach the backside. Then the increased absorbance per layer becomes compensated by the decrease in penetration depth.
Again we compare the dipole results for the total absorbance with the results obtained by means of Fresnel. To this end we introduce the proportional absorbance as:

Prop. Abs. $=\frac{A_{\mathrm{F}}-A_{\mathrm{DIP}}}{A_{\mathrm{F}}+A_{\mathrm{DIP}}} \times 2$,

where $A_{\mathrm{F}}$ means the Fresnel absorbance and $A_{\text {DIP }}$ the discrete dipole absorbance. This proportional absorbance is given in fig. 7 . The deviations are of the order of magnitude of $10^{-3}$.

As a last comparison between classical continuum and discrete dipole description, we have calculated the transmittance of slabs as a function of the thickness. The results have been collected in table 3 for SAC and a frequency of

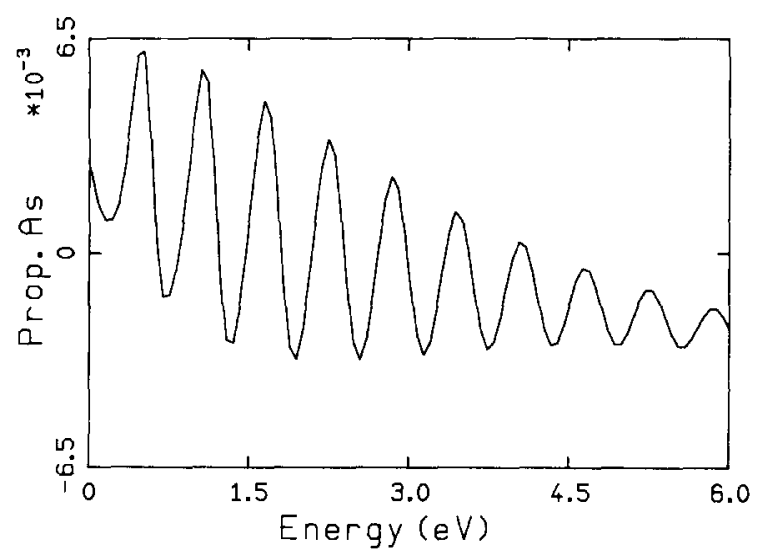

Fig. 7. Comparison with Fresnel: proportional absorbance (see text) of a slab of 509 layers as a function of $\hbar \omega$. SAC-case, $\theta_{i}=0$.

Table 3

Thick slab transmittance.

\begin{tabular}{rll}
\hline Layers & Discrete dipole model & Proportional difference \\
\hline 1 & $9.038479 \times 10^{-1}$ & $6.2 \times 10^{-2}$ \\
3 & $8.005526 \times 10^{-1}$ & $6.2 \times 10^{-2}$ \\
9 & $4.802702 \times 10^{-1}$ & $5.7 \times 10^{-2}$ \\
19 & $2.483535 \times 10^{-1}$ & $2.9 \times 10^{-2}$ \\
49 & $3.247595 \times 10^{-1}$ & $1.9 \times 10^{-2}$ \\
109 & $1.902889 \times 10^{-1}$ & $0.3 \times 10^{2}$ \\
209 & $7.483919 \times 10^{-2}$ & $1.6 \times 10^{-2}$ \\
509 & $9.281507 \times 10^{-3}$ & $1.7 \times 10^{-2}$ \\
1009 & $2.330313 \times 10^{-4}$ & $1.9 \times 10^{-2}$ \\
2009 & $1.454921 \times 10^{-7}$ & $2.4 \times 10^{-2}$ \\
3009 & $9.080240 \times 10^{-11}$ & $2.9 \times 10^{-2}$ \\
\hline
\end{tabular}


$5.0 \mathrm{eV}$. The first column gives the number of layers, the second the transmittance according to the discrete dipole model and the third the proportional difference, defined analogously as in eq. (47). The first 4 rows have been calculated using the thin slab method, the others using the thick slab method with $N_{\mathrm{S}}=13$. The largest discrepancies can be found for the thinnest slabs, which is obvious in view of the nonclassical behaviour of the outermost layers. Until here only perpendicular incidence cases have been studied. In figs. 8 and 9 we also show results as a function of the angle of incidence. In both fig-

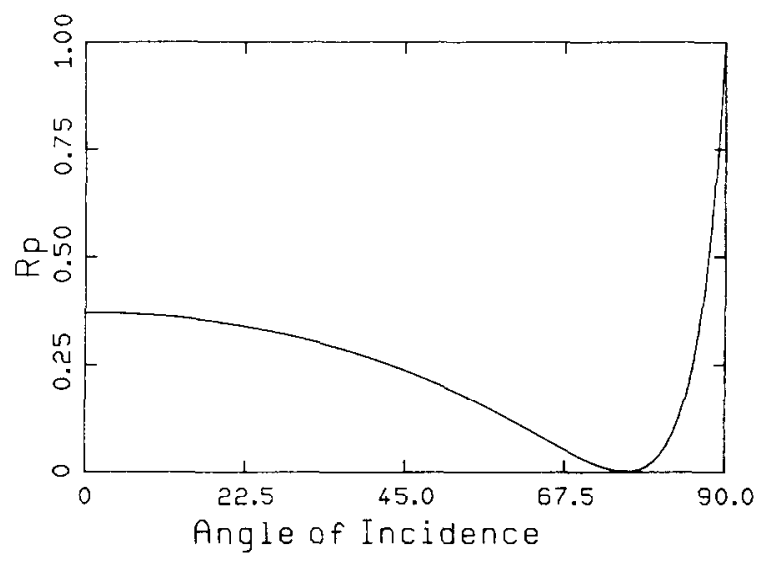

Fig. 8. P-polarized reflectance $R_{p}$ of a slab of 509 layers as a function of $\theta_{i}$. SAC-case, $\hbar \omega=5.0 \mathrm{eV}$.

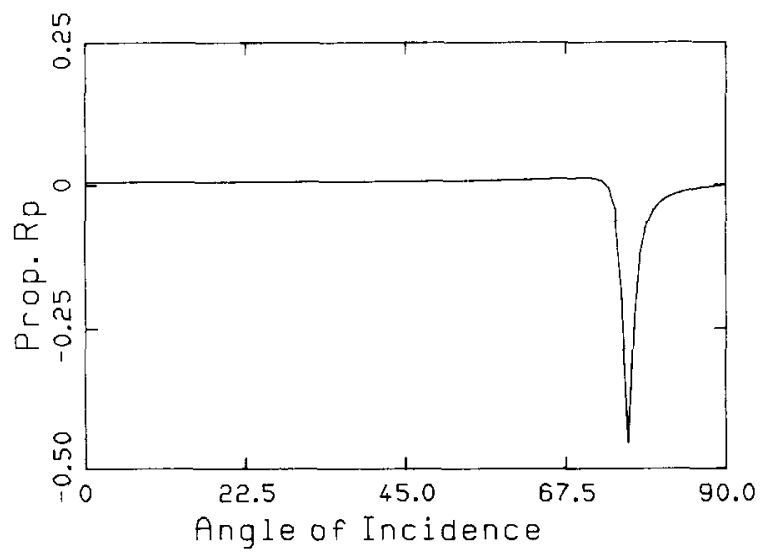

Fig. 9. Comparison with Fresnel: proportional reflectance (see text), P-polarization, as a function of $\theta_{i}$. SAC-case, $\hbar \omega=5.0 \mathrm{eV}$. ures the absorbing strong coupling case has been used for a frequency of $5.0 \mathrm{eV}$.

The p-polarized reflectance for a 509 layer slab has been shown in fig. 8 . The Brewster minimum is strong, indicating that the absorption is still rather modest. Figure 9 shows the proportional difference (analogous to (47)), with respect to the Fresnel calculation. The maximum discrepancy can be found near the Brewster angle. Essentially the same conclusion has been obtained in ref. [21].

\section{Discussion and conclusions}

We have shown in this article that the optical response of thick films (slabs) can be calculated exactly with a full microscopic model also. The hybrid thick slab method where in the surface normal direction real and transformed (normal modes) space approaches have been merged, has been devised for this purpose. The thick slab method has increased the size range for slab calculations, as for instance the thin slab method of Wijers and Del Sole [8], to such an extent, that realistic problems can be studied. This has been achieved without a loss of performance. From about 13 layer slabs on, both memory and CPU-time requirements stabilize, whereas for the more straightforward thin slab method those requirements continue to increase with the square and the cube power of the number of layers, respectively.

Besides this, the model has also clear conceptual advantages. The seemingly contradictory increment of dipole strengths in the direction of propagation, as observed originally for thin slabs, could unambiguously be attributed to a bulk-like interference phenomenon. Again surface local field effects could be observed, as becomes especially clear from a comparison with classical Fresnel calculations. Advantages of this technique will become especially clear if surface microscopic phenomena are going to be investigated optically using transmission through transparent plates. For the interpretation of this class of experiments the thick slab method offers the required combination of microscopic accuracy 
and capability of handling macroscopic sizes. The characteristic parameters determining the model involve the microscopic geometry, the coupling rate $\alpha / \alpha_{0}$ and the energy of the incoming light. At the surfaces, full flexibility as to those parameters exists, e.g. for the incorporation of a surface monolayer of deviating composition. Contrary to a Fresnel-type of approach, where in those cases the method itself has to be changed, the thick slab method asks only for different input parameters. In a forthcoming article where we will focus upon application to experimental work (the optics of an adsorbed monolayer of gas) these options will be fully exploited.

\section{Appendix}

In the derivation of eq. (34) we have used explicitly the orthogonality of the vectors $\boldsymbol{g}_{m}$ and $\boldsymbol{g}_{S, P}$ and their reflected counterparts. Essential for this orthogonality are the projection properties of the tensor $\boldsymbol{d}_{00}$. Suppose we are dealing with an arbitrary vector $\boldsymbol{x}$ which can be written in the $(\hat{\boldsymbol{s}}, \hat{\boldsymbol{p}}, \hat{\boldsymbol{k}})$ reference frame as:

$\boldsymbol{x}=a \hat{\boldsymbol{s}}+b \hat{\boldsymbol{p}}+c \hat{\boldsymbol{k}}$.

From eq. (9) we obtain the explicit expression for $\boldsymbol{d}_{00}$ :

$\boldsymbol{d}_{00}=\frac{2 \pi \mathrm{i}}{\alpha_{0} \beta\left|k_{z}\right|}\left(k^{2} \mathbf{1}-\boldsymbol{k} \boldsymbol{k}^{\mathrm{T}}\right)$.

This tensor becomes diagonal in the $(\hat{\boldsymbol{s}}, \hat{\boldsymbol{p}}, \hat{\boldsymbol{k}})$ frame and it is not difficult to see that the effect of $\boldsymbol{d}_{00}$ upon $\boldsymbol{x}$ becomes:

$\boldsymbol{d}_{00} \boldsymbol{x}=\frac{2 \pi \mathrm{i}}{\boldsymbol{\alpha}_{0} \beta\left|k_{z}\right|}\left(k^{2}(a \hat{s}+b \hat{\boldsymbol{p}})+c\left(k^{2} \hat{\boldsymbol{k}}-\left(\boldsymbol{k} \boldsymbol{k}^{\mathrm{T}}\right) \hat{\boldsymbol{k}}\right)\right)$.

And using $\left(a b^{\mathrm{T}}\right) c=a\left(b^{\mathrm{T}} c\right)$,

$\boldsymbol{d}_{00} \boldsymbol{x}=\frac{2 \pi \mathrm{i} k^{2}}{\alpha_{0} \beta\left|k_{z}\right|}(a \hat{s}+b \hat{p})$.
So it turns out that $\boldsymbol{d}_{00}$ projects any vector $\boldsymbol{x}$ onto the $(\hat{s}, \hat{p})$-plane. This restricts the orthogonality of the $g$-vectors mentioned at the beginning, to the $(\hat{s}, \hat{p})$-plane, since all these $g$-vectors contain $\boldsymbol{d}_{00} \boldsymbol{u}_{m}$ as their essential part. It turns out that in our case, because of (22) all $\boldsymbol{u}_{m}$ can be chosen as follows: $\boldsymbol{u}_{1}, \boldsymbol{u}_{3}$ in the $(\hat{s}, \hat{k})$-plane and $\boldsymbol{u}_{2}, \boldsymbol{u}_{4}$ in the $(\hat{p}, \hat{k})$-plane. Since $\hat{\boldsymbol{s}}$ and $\hat{\boldsymbol{p}}$ are orthogonal the disappearance of matrix elements in (34) has been explained.

\section{References}

[1] R. Rydberg, Phys. Rev. Lett. 49 (1982) 1579

[2] W. Chen and W.L. Schaich, Surf. Sci. 218 (1989) 580.

[3] H.H. Richardson, H.C. Chang, C. Noda and G.E. Ewing, Surf. Sci. 216 (1989) 93.

[4] Y.J. Chabal, Surf. Sci. Rep. 8 (1988) 211, and references therein.

[5] G.P.M. Poppe and C.M.J. Wijers, Proc. 10th Coll. Hertzian Optics and Dielectrics, Rennes (1989).

[6] G.P.M. Poppe and C.M.J. Wijers, to be published.

[7] C.M.J. Wijers and K.M.E. Emmet, Phys. Scripta 38 (1988) 435.

[8] C.M.J. Wijers and R. Del Sole, Phys. Scripta T 25 (1989) 325.

[9] O. Litzman and P. Rózsa, Surf. Sci. 66 (1977) 542.

[10] P.P. Ewald, Ann. Phys. 49 (1916) 117; 54 (1917) 519, 557.

[11] C.M.J. Wijers, Proc. 18th ann. Int. Symp. DSTP, Dresden (GDR) (1989) p. 123.

[12] P. Dub and O. Litzman, Scripta Fac. Sci. Nat. Univ. Purk. Brun. 13 (1983) 283.

[13] O. Litzman, Optica Acta 27 (1980) 231.

[14] B.M.E. van der Hoff and G.C. Benson, Can. J. Phys. 31 (1953) 1087, and references therein.

[15] J. Vlieger, Physica 64 (1973) 63.

[16] J.D.E. McIntyre and D.E. Aspnes, Surf. Sci. 24 (1971) 417.

[17] F.H.P.M. Habraken, O.L.J. Gijzeman and G.A. Bootsma, Surf. Sci. 96 (1980) 482.

[18] N. Kar and A. Bagchi, Solid State Commun. 31 (1980) 645.

[19] W.L. Mochán and R.G. Barrera, Phys. Rev. Lett. 55 (1985) 1192.

[20] M. Born and E. Wolf, Principles of Optics (Pergamon, Oxford, 1985).

[21] C.M.J. Wijers and G.P.M. Poppe, Proc. IVC-11/ICSS7, Cologne 1989 , to be published as a regular issue of Vacuum. 